\title{
Solvability of a second-order differential equation with integral boundary condition at resonance on an unbounded domain
}

\section{XiAOJIE LiN, ZENGJi DU and QIN ZHANG}

\begin{abstract}
.
This paper deals with the solvability of two class of second-order integral boundary value problems at resonance on a half-line. Both of the boundary value conditions are responsible for resonance. By using the coincidence degree theory, we establish a new general existence result.
\end{abstract}

Acknowledgements. This work is supported by the Natural Science Foundation of China (Grant No. 11071205 and 11101349), and partially supported by the Natural Science Foundation of Jiangsu Province (Grant No. BK2011042), the Natural Science Foundation of Jiangsu Education Department (11KJB110013) and Qing Lan Project.

\section{REFERENCES}

[1] Agarwal, R. P. and O'Regan, D., Infinite Interval Problems for Differential, Difference and Integral Equations, Kluwer Academic, 2001

[2] Feng, W. and Webb J. R. L., Solvability of three-point boundary value problems at resonance, Nonlinear Anal., 30 (1997), 3227-3238

[3] Gupta, C. P., A second order m-point boundary value problem at resonance, Nonlinear Anal., 24 (1995), 1483-1489

[4] Kosmatov, N., Multi-point boundary value problems on an unbounded domain at resonance, Nonlinear Anal., 68 (2008), 2158-2171

[5] Kosmatov, N., Second order boundary value problems on an unbounded domain, Nonlinear Anal., 68 (2008), 875-882

[6] Lian, H. and Ge, W., Solvability for second-order three-point boundary value problems on a half-line, Appl. Math. Lett., 19 (2006), 1000-1006

[7] Lian, H., Pang, H. and Ge, W., Solvability for second-order three-point boundary value problems at resonance on a half-line, J. Math. Anal. Appl., 337 (2008), 1171-1181

[8] Ma, R., Multiplicity results for a third order boundary value problem at resonance, Nonlinear Anal., 32 (1998), 493-499

[9] Ma, R., Existence of positive solution for second-order boundary value problems on infinite intervals, Appl. Math. Lett., 16 (2003), 33-39

[10] Mawhin, J., Topological degree methods in nonlinear boundary value problems, NSFCBMS, Regional Conference Series in Mathematics, American Mathematical Society, Providence, RI, 1979

JIANGSU NORMAL UNIVERSITY

SCHOOL OF MATHEMATICS AND STATISTICS

XUZHOU, JIANGSU, 221116, P. R. CHINA

E-mail address: Iinxiaojie1973@163.com

E-mail address: duzengji@163.com

E-mail address: zhangqininxznu@yahoo.com.cn.

Received: 29.01.2011; In revised form: 04.05.2012; Accepted: 24.09.2012

2010 Mathematics Subject Classification. 34B15.

Key words and phrases. Integral boundary value problem, coincidence degree theory, resonance, unbounded domain. Corresponding author: Zengji Du; duzengji@163.com 